\title{
ON INSCRIBING $n$-DIMENSIONAL SETS IN A REGULAR $n$-SIMPLEX
}

\section{DAVID GALE}

1. Introduction. It was proved in 1901 [1] that every set of diameter 1 in $n$-space can be inscribed in an $n$-sphere of radius $r$ $\leqq(n / 2(n+1))^{1 / 2}$. The best and most recent proof was given in 1941 [2]. The geometric content of this result is made apparent by the following equivalent formulation: the circumscribed $n$-sphere of a regular $n$-simplex of diameter 1 will cover any $n$-dimensional set of diameter 1.

In this paper we prove a dual to the above result, namely (Theorem 1): the regular $n$-simplex whose inscribed sphere has diameter 1 will cover any $n$-dimensional set of diameter 1 . We then apply this result to the case $n=2$ to show that every plane set of diameter 1 is the union of three sets each of diameter less than $3^{1 / 2} / 2$. This result is related to the still unsolved conjecture of $K$. Borsuk [3] that every set in $n$-space of diameter 1 is the union of $n+1$ sets each of diameter less than 1. Our result gives the strongest possible answer to this question for $n=2$.

\section{Main theorem.}

Notation. Throughout this paper lower case letters will denote vectors, Greek letters will denote (real) scalars, the scalar product of the vectors $x$ and $y$ will be denoted by $x y$, and the norm of a vector $x$ by $|x|$.

Definitions. By an $n$-simplex $\Sigma$ in a Euclidean space we shall mean the convex closure of a set of $n+1$ vectors $v_{0}, v_{1}, \cdots, v_{n}$. We shall say that $\Sigma$ is regular if the numbers $\left|v_{i}-v_{j}\right|$ are equal for $i \neq j$, $i, j=0, \cdots, n$. An $(n-1)$-face of $\Sigma$ is the convex hull of any $n$ vectors from among the $v_{i}$. A set $S$ is said to be inscribed in $\Sigma$ if $S \subset \Sigma$ and $S$ intersects every $(n-1)$-face of $\Sigma$.

We now state the principal result.

TheOREM I. Let $S$ be a closed subset of $n$-space of diameter 1 . Then $S$ can be inscribed in a regular $n$-simplex of diameter $d \leqq(n(n+1) / 2)^{1 / 2}$.

Proof. Let $V$ denote $(n+1)$-dimensional Euclidean space with unit vectors $e_{0}, e_{1}, \cdots, e_{n}$. Let $\bar{e}=\sum_{i=0}^{n} e_{i}$ and let $L=\{x \mid x \in V$, $x \bar{e}=0\}$. Since $L$ is an $n$-dimensional linear subspace of $V$ we may assume that $S$ is imbedded in $L$.

Presented to the Society, September 5, 1952; received by the editors June 11, 1952. 
Let $\alpha_{i}=\min _{x \in S} x e_{i}$, and let $a=\sum_{i=0}^{n} \alpha_{i} e_{i}$.

Let $\beta_{i}=\max _{x \in s} x e_{i}$, and let $b=\sum_{i=0}^{n} \beta_{i} e_{i}$.

Let $H_{i}^{-}=\left\{x \mid x e_{i} \geqq \alpha_{i}\right\}, H_{i}^{+}=\left\{x \mid x e_{i} \leqq \beta_{i}\right\}$.

Let $\Sigma^{-}=\bigcap_{i=0}^{n} H_{i}^{-} \cap L, \Sigma^{+}=\bigcap_{i=0}^{n} H_{i}^{+} \cap L$.

We shall show that $\Sigma^{-}$and $\Sigma^{+}$are regular $n$-simplexes and at least one of them has diameter $d \leqq(n(n+1) / 2)^{1 / 2}$. The proof will be divided into three parts.

(1) We first show that $a \bar{e} \leqq 0$ and $a \bar{e}=0$ only if $S$ consists of a single point.

For any $x \in S, x e_{i} \geqq \alpha_{i}$, hence $\sum_{i=0}^{n}\left(x e_{i}\right)=x \bar{e} \geqq \sum_{i=0}^{n} \alpha_{i}=a \bar{e}$, but $x \in L$ so $x \bar{e}=0$, hence $a \bar{e} \leqq 0$. If $a \bar{e}=0$ then $\sum_{i=0}^{n} \alpha_{i}=0$ and for any $x \in S, \quad \sum_{i=0}^{n} x e_{i}=0$, but $x e_{i}-\alpha_{i} \geqq 0$, so $\sum_{i=0}^{n}\left(x e_{i}-\alpha_{i}\right)=0$ implies $x e_{i}=\alpha_{i}$ for all $i$, hence $x=a$. Similarly one shows that $b \bar{e} \geqq 0$ and equality holds only if $S$ consists of a single point. Since in the latter case the theorem is trivial we assume henceforth that $a \bar{e}<0$ and $b \bar{e}>0$.

(2) $\Sigma^{-}$and $\Sigma^{+}$are $n$-simplexes and $S$ is inscribed in both.

We shall show that $\Sigma^{-}$is the simplex spanned by the vectors $v_{0}, v_{1}, \cdots, v_{n}$ where $v_{i}=a-(a \bar{e}) e_{i}$. First suppose $x=\sum_{i=0}^{n} \lambda_{i} v_{i}$ where $\lambda_{i} \geqq 0$ and $\sum_{i=0}^{n} \quad \lambda_{i}=1$. Then $x=\sum_{i=0}^{n} \quad \lambda_{i}\left(a-(a \bar{e}) e_{i}\right)=a$ $-(a \bar{e}) \sum_{i=0}^{n} \lambda_{i} e_{i}$, so $x e_{i}=\alpha_{i}-\lambda_{i}(a \bar{e}) \geqq \alpha_{i}$ since $a \bar{e}<0$. Also $x \bar{e}=\sum_{i=0}^{n} x e_{i}$ $=\sum_{i=0}^{n} \alpha_{i}-a \bar{e}=0$, so $x \in \Sigma^{-}$.

On the other hand suppose $x \in \Sigma^{-}$. Then $x=\sum_{i=0}^{n} \mu_{i} e_{i}$ where $\mu_{i}=x e_{i} \geqq \alpha_{i}$ and $\sum_{i=0}^{n} \mu_{i}=0$. Now since $a \bar{e} \neq 0$ we can write $e_{i}$ $=(1 / a \bar{e})\left(a-v_{i}\right)$, so $x=(1 / a \bar{e}) \sum_{i=0}^{n} \quad \mu_{i}\left(a-v_{i}\right)=-(1 / a \bar{e}) \sum_{i=0}^{n} \mu_{i} v_{i}$. From the definition of $v_{i}$ we see that $\sum_{i=0}^{n} \alpha_{i} v_{i}=0$, so we may write

$$
x=-(1 / a \bar{e}) \sum_{i=0}^{n} \mu_{i} v_{i}+(1 / a \bar{e}) \sum_{i=0}^{n} \alpha_{i} v_{i}=(1 / a \bar{e}) \sum_{i=0}^{n}\left(\alpha_{i}-\mu_{i}\right) v_{i},
$$

and $\left(\alpha_{i}-\mu_{i}\right) / a \bar{e} \geqq 0$ since $\mu_{i} \geqq \alpha_{i}$ and $a \bar{e}<0$. Also $(1 / a \bar{e}) \sum_{i=0}^{n}\left(\alpha_{i}-\mu_{i}\right)$ $=\sum_{i=0}^{n} \alpha_{i} / a \bar{e}=1$. Thus $\Sigma-$ is the convex closure of the $v_{i}^{\prime}$ 's.

To show that $S$ is inscribed in $\Sigma$ - we must show that it has a point in common with each $(n-1)$-face of $\Sigma^{-}$. Now there is an $x \in S$ such that $x e_{i}=\mu_{i}=\alpha_{i}$. Using this in the expression $\left({ }^{*}\right)$ above we see that $x$ lies in the $(n-1)$-face of $\Sigma$ - opposite the vertex $v_{i}$.

The proof for $\Sigma^{+}$is similar.

(3) $\Sigma^{-}$and $\Sigma^{+}$are regular simplexes of diameter $d^{-}=-2^{1 / 2} a \bar{e}$ and $d^{+}=2^{1 / 2} b \bar{e}$ respectively, and $\min \left(d^{-}, d^{+}\right) \leqq(n(n+1) / 2)^{1 / 2}$.

Working with $\Sigma^{-}$, for $i \neq j$ we have $v_{i}-v_{j}=-a \bar{e}\left(e_{i}-e_{j}\right)$ so $\left|v_{i}-v_{j}\right|$ $=\left((a e)^{2}\left(e_{i}-e_{j}\right)^{2}\right)^{1 / 2}=2^{1 / 2}|a \bar{e}|=-2^{1 / 2} a \bar{e}=d^{-}$, and similarly each edge of $\Sigma^{+}$has length $d^{+}=2^{1 / 2} b \bar{e}$. To estimate min $\left(d^{-}, d^{+}\right)$we first show that $\beta_{i}-\alpha_{i} \leqq(n /(n+1))^{1 / 2}$ for all $i$. It suffices to show this for $\beta_{0}-\alpha_{0}$. Choose $x$ and $y \in S$ such that $x e_{0}=\alpha_{0}, y e_{0}=\beta_{0}$ and let $x=\alpha_{0} e_{0}$ 
$+\sum_{i=1}^{n} \gamma_{i} e_{i}, y=\beta_{0} e_{0}+\sum_{i=1}^{n} \delta_{i} e_{i}$ and let $\theta_{0}=\beta_{0}-\alpha_{0}, \theta_{i}=\delta_{i}-\gamma_{i}$ for $i>0$. Then $\sum_{i=0}^{n} \theta_{i}=0$ and $\sum_{i=0}^{n} \theta_{i}^{2} \leqq 1$, using for the first time the hypothesis that the diameter of $S$ is 1 . Now $0 \leqq \sum_{i=1}^{n}\left(\theta_{i}+\theta_{0} / n\right)^{2}$ $=\sum_{i=1}^{n} \theta_{i}^{2}+\left(2 \theta_{0} / n\right) \sum_{i=1}^{n} \theta_{i}+\theta_{0}^{2} / n=\sum_{i=1}^{n} \theta_{i}^{2}-\theta_{0}^{2} / n$, so $\theta_{0}^{2} / n \leqq \sum_{i=1}^{n} \theta_{i}^{2}$ and $((n+1) / n) \theta_{0}^{2} \leqq \sum_{i=0}^{n} \theta_{i}^{2} \leqq 1$ which gives $\theta_{0} \leqq(n /(n+1))^{1 / 2}$.

Now $d^{+}+d^{-}=2^{1 / 2}(b \bar{e}-a \bar{e})=2^{1 / 2} \sum_{i=0}^{n}\left(\beta_{i}-\alpha_{i}\right) \leqq(2 n(n+1))^{1 / 2}$ and since $d^{+}$and $d^{-}$are both positive it follows that one of them is less than or equal to $(n(n+1) / 2)^{1 / 2}$ completing the proof.

REMARK. One easily computes the diameter of the regular $n$-simplex whose inscribed sphere has diameter 1 , and finds it to be exactly $(n(n+1) / 2)^{1 / 2}$. Therefore the inequality we have obtained is the best possible.

3. Applications. We use the preceding result to prove the following theorem.

Theorem II. Every plane set $S$ of diameter 1 is the union of 3 sets each of diameter $d \leqq 3^{1 / 2} / 2$.

Proof. By Theorem I, $S$ can be inscribed in an equilateral triangle $\Delta$ of side $s \leqq 3^{1 / 2}$. Assuming the most unfavorable case, i.e., that $s=3^{1 / 2}$, we shall describe the desired decomposition.

Let $\Delta$ have vertices $A, B$, and $C$, let $a$ and $b$ be the midpoints of $B C$ and $A C$ respectively, and let $O$ be the center of $\Delta$. Let $\gamma$ be a line parallel to and of distance 1 from $A B$, intersecting $A C$ and $B C$ in points $p$ and $q$ respectively. Since the diameter of $S$ is $1, S$ lies between lines $\gamma$ and $A B$. Let $R_{1}$ be the pentagon with vertices $O b p q a$ and let $R_{2}$ and $R_{3}$ be the region obtained by rotating $R_{1}$ about $O$ through angles $2 \pi / 3$ and $4 \pi / 3$. Then $S \subset R_{1} \cup R_{2} \cup R_{3}$. By direct computation one shows that the diameter of $R_{1}$ is the distance from $a$ to $b$ which is $3^{1 / 2} / 2$, and since $R_{2}$ and $R_{3}$ are congruent to $R_{1}$ the theorem is proved.

REMARK. It is easily shown that a disc of diameter 1 cannot be decomposed into three sets each having diameter less than $3^{1 / 2} / 2$ and therefore the inequality of Theorem $I$ is the best possible. This result suggests a strengthening of the Borsuk conjecture, as follows. Let the $n$-sphere of diameter 1 be decomposed into $n+1$ sets, $S_{0}, S_{1}, \cdots, S_{n}$ so that $\max _{i}$ (diameter $S_{i}$ ) $=d_{n}$ where $d_{n}$ is as small as possible. Then every set of diameter 1 in $n$-space is the union of $n+1$ sets of diameter at most equal to $d_{n}$.

As a further application of the main theorem one can prove the known fact [4] that every plane set of diameter 1 can be imbedded in a regular hexagon the distance between whose opposite sides is 1 . 
In three-space one can show that every set of diameter 1 can be imbedded in a regular octahedron the distance between whose opposite faces is 1 . We omit the proofs.

\section{BIBLIOGRAPHY}

1. H. W. E. Jung, Über die kleinste kugel, die eine räumliche Figur einschliesst, J. Reine Angew. Math. vol. 123 (1901) pp. 241-257.

2. L. M. Blumenthal and G. E. Wahlin, On the spherical surface of smallest radius enclosing a bounded subset of $n$-dimensional Euclidean space, Bull. Amer. Math. Soc. vol. 47 (1941) pp. 771-777.

3. K. Borsuk, Drei Sätze ïber die n-dimensionale euklidische Sphäre, Fund. Math. vol. 20 (1933) pp. 177-190.

4. T. Bonnesen and W. Fenchel, Theorie der konvexen Körper, Springer, 1934, pp. 130-131.

BROWN UNIVERSITY 